\title{
WARP: a double phase Argon programme for Dark Matter detection
}

\author{
E. Calligarich, M. Cambiaghi, C. De Vecchi, R. Dolfini, L. Grandi, A. Menegolli, \\ C. Montanari, M. Prata, A. Rappoldi, G.L. Raselli,M. Roncadelli, M. Rossella, \\ C. Rubbia;, C. Vignoli
}

INFN and Department of Physics at University of Pavia

Via Bassi 6 - I27100 Pavia (Italy)

\section{F. Carbonara, Alfredo G. Cocco ${ }^{\dagger}$ A. Ereditato, G. Fiorillo, G. Mangano, R. Santorelli INFN and Department of Physics at University of Napoli "Federico II" \\ Via Cintia - I80126 Napoli (Italy)}

\section{F. Cavanna, N. Ferrari, O. Palamara}

INFN Laboratori Nazionali del Gran Sasso and University of L'Aquila

SS 17 bis km 18+910 - I67010 L'Aquila (Italy)

\section{Galbiati}

Department of Physics - Princeton University

Princeton, NJ - 08544 (USA)

\section{A. Szelc}

Institute of Nuclear Physics - PAN

ul. Radzikowskiego 152, 31-342 Krakow (Poland)

\begin{abstract}
The WARP (WIMP Argon Programme) experiment aims to the detection of WIMP elastic scattering interactions occurring in a target of liquid Argon. The simultaneous detection of prompt scintillation light in liquid Argon and delayed signals coming form the extraction of ionization electrons in the gas phase provides a clean identification of recoil events in the range $10 \div 100$ $\mathrm{keV}$. The 100 liter detector under construction at the Laboratori Nazionali del Gran Sasso (Italy) will be able to achieve a sensitivity of few $10^{-8}$ pbarn. In the same underground site a 2.3 liter prototype has been operated for one year collecting more than $5 \times 10^{6}$ events. Based on this data, results on the amount of ${ }^{39} \mathrm{Ar}$ isotope in commercial liquid Argon together with a study on the background rejection power achievable by the event selection technique is reported.
\end{abstract}

International Europhysics Conference on High Energy Physics

July 21st - 27th 2005

Lisboa, Portugal

\footnotetext{
${ }^{*}$ Spokesperson

${ }^{\dagger}$ Speaker.

$\ddagger$ supported by a Grant of the President of the Polish Academy of Sciences
} 


\section{Introduction}

Recent discoveries in cosmology, astrophysics and particle physics seem to indicate that only $5 \%$ of our Universe is made of ordinary matter and visible electromagnetic radiation.Observations on the galaxies rotation curves, gravitational lensing and large scale structure could be explained assuming that a large fraction of the Universe (as much as 30\%) should be made of neutral, non relativistic and non baryonic matter, the so called Dark Matter. Elementary particle physics offers a solution to this problem by assuming that this matter could be constituted by weakly interacting massive particles (WIMPs); the most promising WIMP candidate is the Lightest Super-symmetric Particle (LSP) found in Super-symmetric extensions of the Standard Model of Unified interactions. According to this model and to recent experimental results the WIMP-nucleus interaction cross section is smaller than $10^{-6}$ pbarn; the experimental sensitivity is thus limited by the background conditions and the active detector mass. The background can be reduced by going deep underground in order to shield the detector from cosmic rays and by adding a shield against natural radioactivity. Detector mass is limited by the choice of the detection technique and of the target nucleus. The WARP experiment [1] has been conceived profiting from the experience achieved by the ICARUS [2] collaboration in the use of cryogenic noble liquids; liquid Argon operated in the so called "double phase" technique has proven to be an excellent solution to the problem of having a large detector mass together with a very low detection threshold and background.

\section{The WARP experiment}

The WARP experiment aims at the discovery of WIMPs via the simultaneous detection of scintillation light and ionization produced by WIMPs interacting in a 100 liter volume of liquid Argon [1]. Due to the small energy threshold (about $19 \mathrm{eV}$ ) Liquid Argon has been known since a long time as an excellent scintillator ( $\mathrm{Ar}_{2}$ dimer de-excite at a typical wavelength of $128 \mathrm{~nm}$ ). To collect also the ionization electrons produced in the interaction, an electric field of $1 \mathrm{kV} / \mathrm{cm}$ is generated in the target volume and electrons are drifted toward the liquid-gas interface. Electrons are then extracted to the gas phase and drifted in a region where linear multiplication occurs (about 30 $\gamma / \mathrm{cm}$ ) by means of a set of grids placed at appropriate voltage. This scintillation signal is delayed with respect to the interaction occurring in liquid Argon by the time needed by electrons to drift in the liquid phase $(1.5 \mathrm{~mm} / \mu \mathrm{s})$. At the top of the gas phase 612 inches photomultipliers are used to record both primary scintillation light (primary signal) and multiplication light (secondary signal). The total coverage of the PMTs is of about $10 \%$ of the total surface; a wavelength shifter, mainly TetraPhenylButadiene (emission centered around $438 \mathrm{~nm}$ ), is foreseen both on the PMTs and on the inner walls of the detector in order to shift the Ar scintillation light to the sensitivity region of the PMTs. The reason to record both scintillation (S1) and ionization (S2) signals produced in the WIMP scattering with Argon nuclei is that the ratio S2/S1 appear to characterize nuclear recoils with respect to background induced by gamma rays and electrons, as shown in Figure 1. This phenomenon is described in the so-called Box Model [3] and can be explained by the fact that nuclear recoils produce a higher ionization density, enhancing the probability of electron-ion recombination and giving less free electrons to be drifted to the multiplication region. Another discrimination technique is based on the analysis of the the rise-time of the primary signal; as de- 


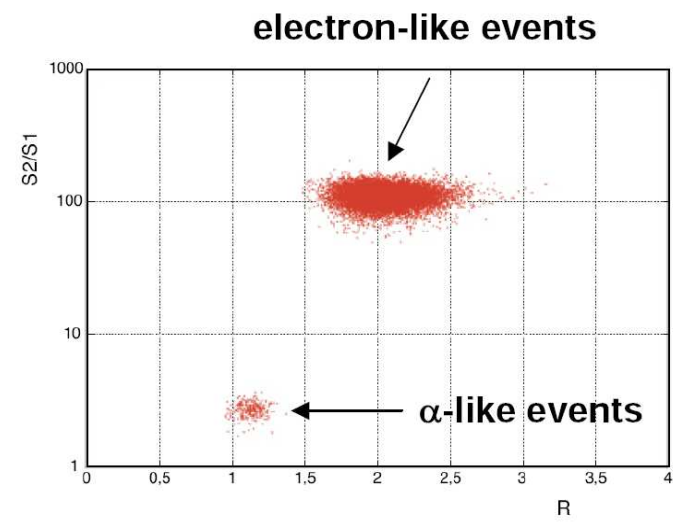

Figure 1: Combined effect of the two background rejection criteria described in the text. The data sample consists of about $2 \times 10^{5}$ events; the separation is clearly visible and no events outside the two regions were found.

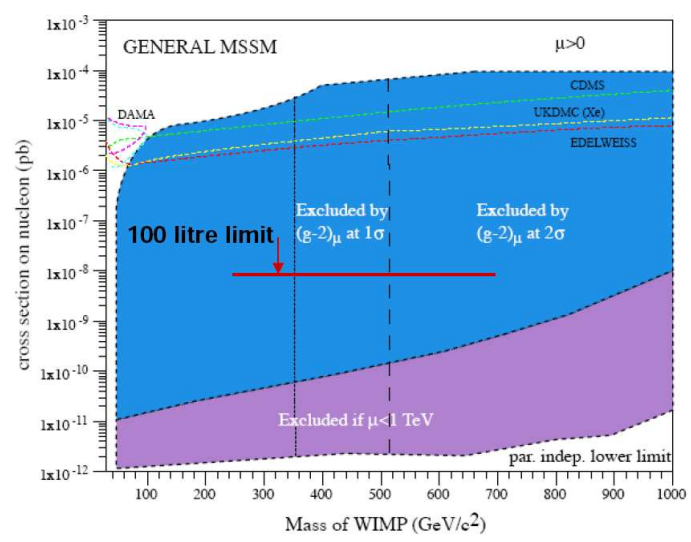

Figure 2: Warp discovery potential represented in the general MSSM plot of spin-independent neutralino nucleon cross-section vs. neutralino mass. The regions excluded by experimental direct searches are also shown at the top of the plot.

scribed in [4] the Argon scintillation light is mainly produced by the de-excitation of the $\mathrm{Ar}_{2}$ dimer singlet $\left({ }^{1} \Sigma\right)$ and triplet $\left({ }^{3} \Sigma\right)$ molecular states. The two states have very different lifetimes $\left(\tau_{1}=7\right.$ ns and $\tau_{3}=1.8 \mu \mathrm{s}$ ) and are populated in a different way by recoil-induced and electron-induced interactions, being $I_{1} / I_{3}=0.3$ for electrons and $I_{1} / I_{3}=1.3 \div 3.0$ for heavy charged particles. A pulse shape analysis has been performed taking the ratio $\mathrm{R}$ between the total pulse height and the fraction of the pulse height in the first $400 \mathrm{~ns}$; preliminary tests made on a set of events collected with a prototype chamber showed that a clear separation is achieved between alpha-induced and electron-induced events. As shown in Figure 1 the two selection criteria are largely uncorrelated and a total background rejection power of $10^{7}$ is expected for the WARP experiment. In Figure 2 the discovery potential of the WARP 100 liter detector is shown.

\section{The 2.3 liter prototype}

A small prototype of the WARP detector has been built and operated in the Laboratori Nazionali del Gran Sasso underground site since February 2005. The detector is shown in Figure 3 and consists of an active volume of 2.3 liters (radius of $10 \mathrm{~cm}$ with a drift distance of about $7.5 \mathrm{~cm}$ ). Two different setups have been tested: in the first one seven 2" PMTs have been used while in the second four 3" PMTs were arranged on the top of the gas phase. The PMTs are manufactured by Electron Tubes Ltd with a special Platinum coating in order to maintain photocathode conductivity also at liquid Argon temperature ( $87 \mathrm{~K}$ ). In both cases a light yield of 1.0 photoelectron/keV was obtained. The PMTs are read by a set of charge sensitive preamplifiers (Camberra Mod.2005) whose output is digitized by $50 \mathrm{MHz}$ sampling ADCs [2]. A total statistic of $5 \times 10^{6}$ events has been recorded up to June 2005. During this test period a shield made of $10 \mathrm{~cm}$ of lead was used in order to reduce external gamma ray induced background. An interesting side result of the preliminary analysis of electron-like events in the low energy window is shown in Figure 4. These events are mainly due 


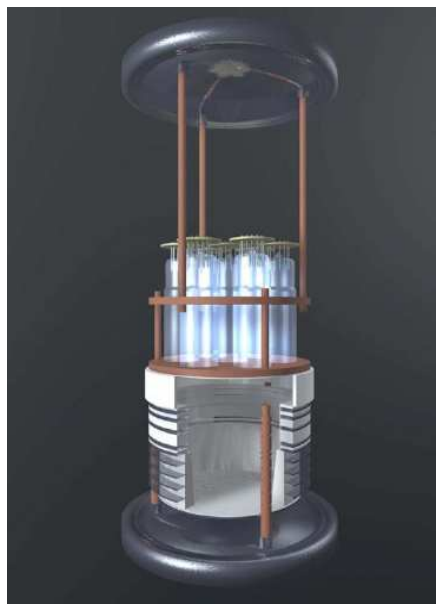

Figure 3: Drawing of the 2.3 liter prototype detector taking data in underground laboratory at Gran Sasso (Italy). The configuration with seven 2 inches PMTs is shown.

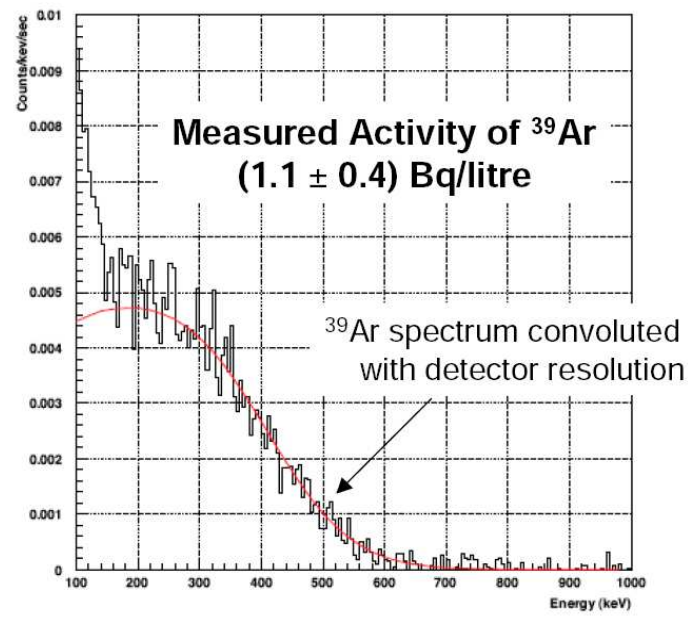

Figure 4: Primary scintillation light for low energy interactions in the 2.3 liter detector prototype. A total ${ }^{39} \mathrm{Ar}$ activity of $1.1 \pm 0.4 \mathrm{~Bq} / \mathrm{l}$ has been measured by the use of MonteCarlo simulated event spectra.

to the beta decay of ${ }^{39} \mathrm{Ar}$, a naturally occurring isotope produced by cosmic rays in the atmosphere. The measured activity is in agreement with the known content of ${ }^{39} \mathrm{Ar}$ in natural Argon reported in [5]. Using this result, a total amount of 3.3 events/s induced by ${ }^{39} \mathrm{Ar}$ beta decay is expected in the WARP 100 liter detector in the energy range $30 \div 100 \mathrm{keV}$. The rejection power of about $10^{7}$, as discussed in the previous paragraph, will reduce this (potential) background to a level of less than one event each 100 days.

\section{Conclusions}

The WARP double phase Argon detector is able to detect the very low energy $(30 \div 100 \mathrm{keV})$ Argon recoils induced by WIMP elastic scattering. The ratio between the primary scintillation light and the secondary signal produced at the multiplication grids togheter with a pulse shape analysis of the primary signal provide a powerful tool to select WIMP induced Argon recoils with respect to the electron-like induced background. The WARP experiment will take data in the second half of 2006 and probe the WIMP-nucleus cross section down to values of about $10^{-8}$ pbarn.

\section{References}

[1] WARP experiment proposal, March 2004 - INFN; Web site:warp.pv.infn.it

[2] S. Amerio et al., Nucl. Inst. Meth. A527 (2004) 329

[3] J. Thomas and D.A. Imel, Phys. Rev. A38 (1988) 5793

[4] A. Hitachi et al., Phys. Rev. B27 (1983) 5279

[5] H.H. Loosli and H. Oeschger, Earth and Plan. Sci. Lett. 7 (1969) 67 